\title{
The STELLA Robotic Observatory on Tenerife
}

\author{
Klaus G. Strassmeier, Thomas Granzer, Michael Weber, Manfred Woche, \\ Emil Popow, Arto Järvinen, Janos Bartus, Svend-Marian Bauer, Frank Dionies, \\ Thomas Fechner, Wilbert Bittner, and Jens Paschke
}

Cosmic Magnetic Fields, Astrophysical Institute Potsdam, An der Sternwarte 16, D-14482 Potsdam, Germany

Correspondence should be addressed to Klaus G. Strassmeier, kstrassmeier@aip.de

Received 23 June 2009; Accepted 5 October 2009

Academic Editor: Alberto J. Castro-Tirado

Copyright ( $\odot 2010$ Klaus G. Strassmeier et al. This is an open access article distributed under the Creative Commons Attribution License, which permits unrestricted use, distribution, and reproduction in any medium, provided the original work is properly cited.

The Astrophysical Institute Potsdam (AIP) and the Instituto de Astrofísica de Canarias (IAC) inaugurated the robotic telescopes STELLA-I and STELLA-II (STELLar Activity) on Tenerife on May 18, 2006. The observatory is located on the Izaña ridge at an elevation of $2400 \mathrm{~m}$ near the German Vacuum Tower Telescope. STELLA consists of two $1.2 \mathrm{~m}$ alt-az telescopes. One telescope fiber feeds a bench-mounted high-resolution echelle spectrograph while the other telescope feeds a wide-field imaging photometer. Both scopes work autonomously by means of artificial intelligence. Not only that the telescopes are automated, but the entire observatory operates like a robot, and does not require any human presence on site.

\section{Introduction}

STELLA is a robotic observatory with two $1.2 \mathrm{~m}$ fully automatic telescopes located at the Teide Observatory in Tenerife, Spain $[1,2]$. Not only the telescopes are automatic but also the entire observatory; no human presence is needed for observing, data reduction, and archiving. The STELLA Echelle spectrograph (SES) on STELLA-I is in robotic operations mode for three years now, and produced approximately 20,000 spectra of the entire optical range between 390 and $900 \mathrm{~nm}$ at a spectral resolution of 55,000 with a peak shutteropen time of $93 \%$ [3]. (The time during a night that is used for science exposures.) Although we do not use an iodine cell nor an actively stabilized chamber or simultaneous ThAr wavelength calibration, its radial velocity precision over the past two years was $30 \mathrm{~m} / \mathrm{s}$ rms depending on target. The Wide-Field STELLA Imaging Photometer (WiFSIP) is currently being tested and is scheduled to enter operation in early 2010 on STELLA-I when the SES fiber will be moved to STELLA-II.

The central scientific objective of STELLA is to understand the structure and dynamics of stellar surface activity and its impact on stellar evolution. The magnetic fields that permeate stellar atmospheres affect the transport of energy and momentum and give rise to surface features similar to the spots and plages we observe on the Sun. These features change with time as a function of the cyclic behaviour of magnetic activity. Reconstructing the spatial distribution of these features from high-resolution spectra-by means of a tomographic technique called Doppler imaging - is still a great challenge and requires simultaneous photometric and spectroscopic observations over the rotation period of a star without significant data gaps. Together with a systematic determination of rotational periods in open clusters as a function of age, time-series Doppler imaging of active stars is the core science program of STELLA.

\section{A Bit of History and Lessons Learned}

Six years of hard work led to the first stellar spectrum taken by STELLA-I from the star $\alpha$ Tauri (K5III) on September 9, 2005. In late May 2006, STELLA-I started its first robotic operation. By mid September 2009 a total of 18,000 echelle spectra were obtained (Figure 1). The STELLA-II telescope is now expected to enter operation with SES in early 2010.

Several problems had and still are to be solved on the way to a functioning observatory (see Granzer et al., this proceedings). The building was, for example, occupied 


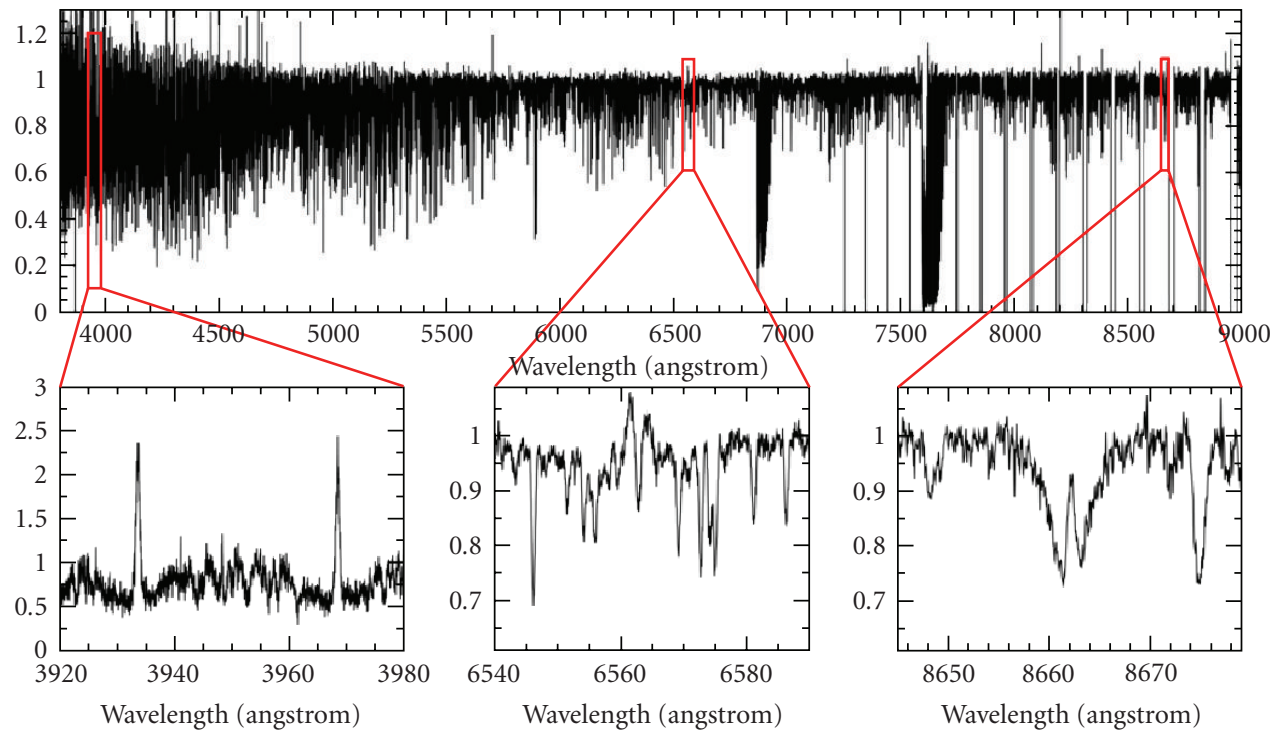

FIgure 1: An example spectrum from the SES. What is shown is the $V=9.0$ mag K0III RS CVn binary XX Triangulum. Exposure time was 1 hour, spectral resolution is 55,000, an $S / N$ ratio of $120: 1$ was achieved. The top panel shows the full spectrum (note the increasing order gaps redwards of $725 \mathrm{~nm}$ ). The lower panels zoom into the Ca II H\&K region, Balmer $\mathrm{H} \alpha$, and one of the Ca II infrared triplets.

by worms which could not find their way out anymore and passed away throughout all the electronics cabinets in the observatory. Chemicals now keep the worms off the building. Frequent ice storms during the winter, as well as one major electromagnetic storm, damaged the building and its electronics. An elaborate control system and a new uninterrupted power supply solve the problem today. Sensors distributed over the entire building continuously report about the environmental conditions inside and outside the building, the telescopes, the scientific instruments, and the many auxiliary systems. The STELLA control system [4-6] linked with an all-sky camera predicts now critical weather conditions such as humidity five minutes in to the future and closes the observatory roof in case of an upcoming storm. A dust monitor was installed just a few months ago to cope with the extremes of the Sahara sand (Calima) and an IR cloud monitor will be installed in early 2010 .

\section{The STELLA Control System (SCS)—Building, Telescope, Instrument, and Data Control and Scheduling}

The basic command structure includes always a command caster and a command listener with precomputed acknowledge times for every task, the latter driven by the hardware response time provided by the various manufacturers. The real advantage of this concept is that it allows for efficient error handling. However, it comes with increased network requirements which are standard though and easily handled with $100 \mathrm{Mbit} / \mathrm{s}$ networks. The principle is laid out in Figure 2. When a command is sent, for example, switch guiding on, a listener receives the command and computes an acknowledge time for that particular task and sends this together with the known execution time to the caster which is waiting for that time to receive the done acknowledge from the listener that, in the meantime, ordered to execute the command or subcommand. Once the listener receives the done from its hardware controller it forwards it to the caster which then decides whether it was done in the precomputed time or not. If not, something went wrong and an error handler is loaded where all messaging is logged. If ok, the caster closes this particular command as being executed successfully. Retrials are done automatically for many but not all processes.

The building is an integrated part and slaved to two redundant weather stations running in coincidence and controlled via the SCS. It was designed to allow full view of the sky for both telescopes at the same time, and to allow emergency closing of the roof at any possible telescope position. This latter feature is a necessity for a secure unattended operation of the system. The roof subsystem is operating autonomously; that is, the power for the roof drives is backed up by a UPS system, and a watchdog makes sure the roof will close if the control computer or the corresponding communication infrastructure fails. All crucial electronics components are backed up by batteries for at least 30 minutes, as are the telescopes. The hydraulic oil pumps for the azimuth bearings are supported by an emergency pressure reservoir, which holds the pressure up until the telescopes come to a complete stop.

Another novel feature is the SCS's weather prediction capability. Meteorology tells us that if the barometric pressure is low the humidity is usually high, and cloudiness is likely. The SCS now learns from the time dependency of the local environmental database how likely this really is. (The database has been built up from the readout of two redundant weather stations with five parameters 


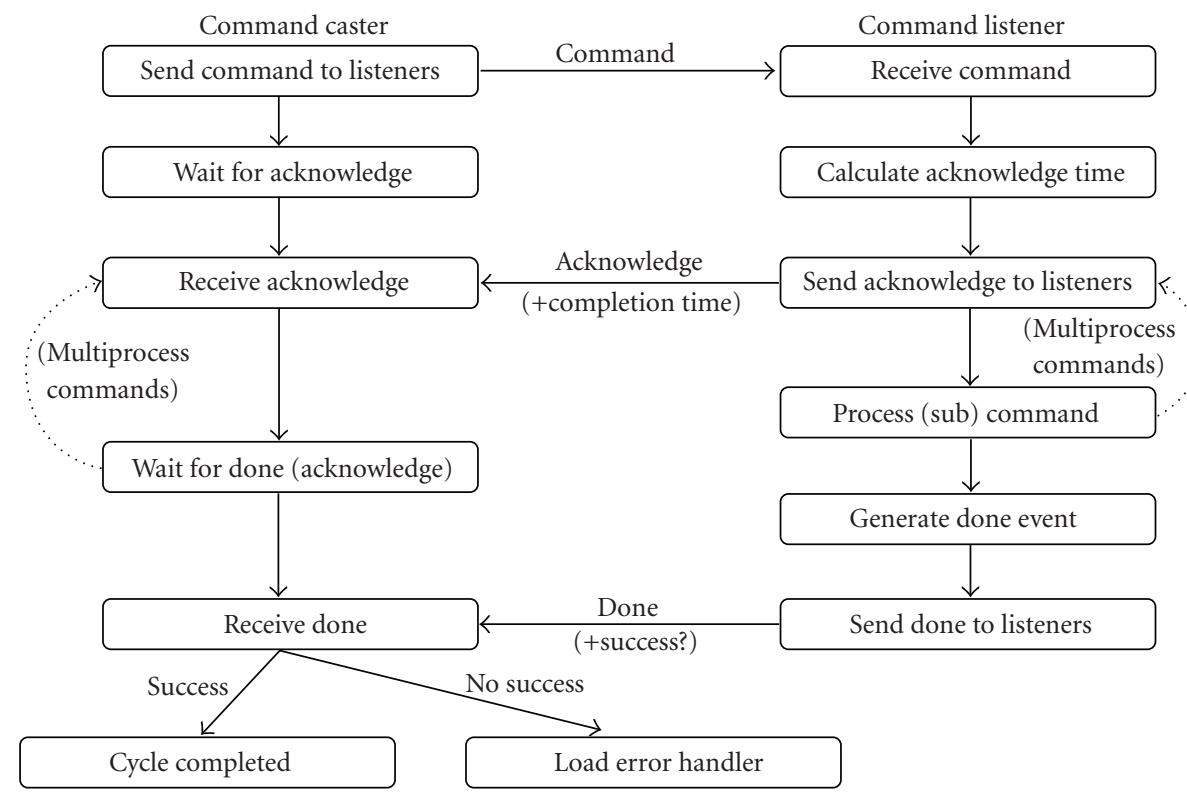

FIGURE 2: The basic messaging system of the STELLA control system (SCS).

each and a full $\log$ is available via a Web interface (see http://www.aip.de/stella).) It predicts the humidity increase from the gradient of the decrease of the barometric pressure and temperature two minutes into the future. Figure 3 depicts an example with various extrapolation curves depending on how many data points and what functionals are used. STELLA is located on the Izaña rim at $2400 \mathrm{~m}$ altitude that first gentle and then steeply falls down to sea level. This allows clouds to creep up from "underneath" and go unnoticed because the all-sky cloud monitor sees clear sky. Fortunately, the clouds announce their coming by increased humidity and, so far, the system did not fail.

We employ a dispatch scheduler for target-load distribution [6]. This is used nowadays on most commercial robotic systems, for example, for air traffic controlling. It runs in real time, allows easy reaction on weather changes, and allows targets to be picked according to actual conditions. The STELLA approach is to split the merit function into a timeslot variable, $t s_{j}$, for long-term merit and a gain variable, $g_{j}$, for short-term merit. The ts variable sets the type of observation, for example, periodic pick, phase-dependent pick, or pick at a predefined time while the $g$ variable sets the immediate priority, for example, by taking into account the air mass during an upcoming integration.

\section{The Telescopes}

Both telescopes were manufactured by Halfmann Teleskoptechnik in Neusäß near Augsburg, Germany (Figure 4(a)). They feature an Alt $/ \mathrm{Az}$ mount and an aperture of $1.2 \mathrm{~m}$. STELLA-I is an $f / 8$ Cassegrain system with two Nasmyth foci and a field of view of $30 \mathrm{arcmin}$. The second telescope (STELLA-II) is equipped with a high-precision spherical mirror with a prime-focus corrector that feeds the optical fiber for the SES at $\mathrm{f} / 8.4$ with a field of view of 2 arcmin. A third telescope, identical to STELLA-I but with an aperture of $0.8 \mathrm{~m}$, is located at the AIP in Potsdam-Babelsberg and serves as a testbed for STELLA instrumentation and interface software as well as for educational purposes (see http://www.aip.de/groups/robotel/).

All telescopes rest on hydrostatic azimuth bearings driven by a single stage oil pump. The altitude axis features a ball bearing. The two telescope axes are moved by direct-drive motors from ETEL with optical Heidenhain encoders. One increment on the encoder corresponds to 36 arcsec, which is interpolated at a resolution of 11 bits, which translates to a theoretical pointing and tracking accuracy of the drives of 0.18 arcsec. If STELLA does not find a target, it assumes that it is not there; that is, no spiral search is done to correct for pointing errors, it then moves on to the next target. After three consecutive failures a set of predefined bright targets distributed over the sky will be moved at and, if not found, the telescope goes to sleep for 30 minutes and then retrials if the weather station permits. We have not recorded a case yet where this was not due to clouds.

The acquisition and guiding unit (AG unit) of a robotic telescope is naturally more complex than for a manual telescope. Guiding on STELLA-I is currently done using a grey beam splitter in the AG-unit. The back-reflected light from an aluminized glass plate with a pinhole for the fiber is simultaneously imaged onto the guiding camera, which allows an estimate of the amount of light reaching the fiber. The diameter of the pinhole is $120 \mu \mathrm{m}$, which corresponds to approximately $2.5 \operatorname{arcsec}$ on the sky. The guiding images are recorded on disk and, after the exposure, averaged and archived for later review if needed. Figure 4 shows the accumulated repositioning of the telescope during a 1-hour stellar integration on a $50 \mu \mathrm{m}$ diameter core of the SES fiber.

Focusing of the telescope is done using a focusing pyramid. At the beginning of operation, a relationship between 


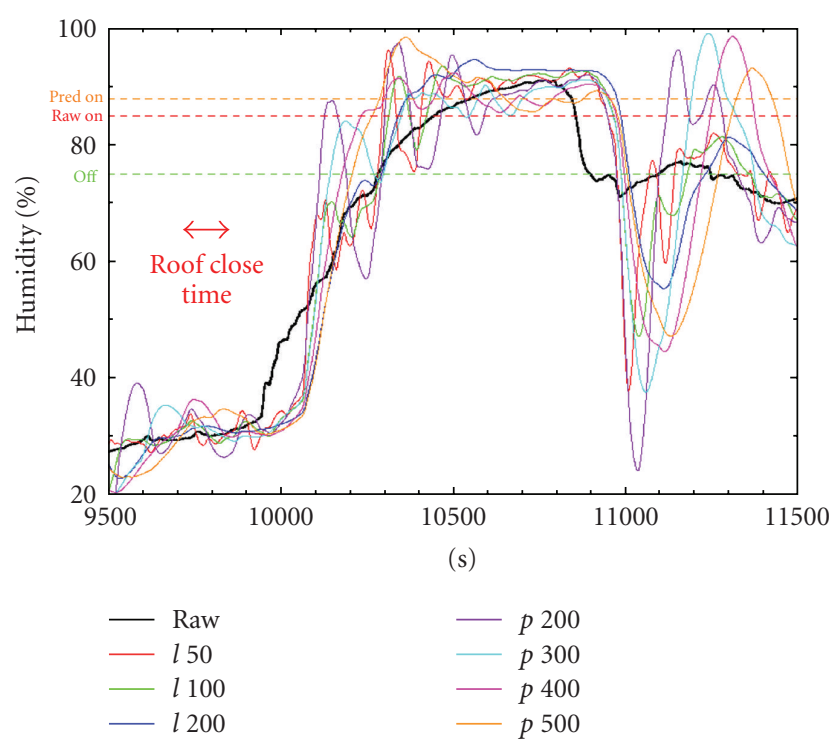

(a)

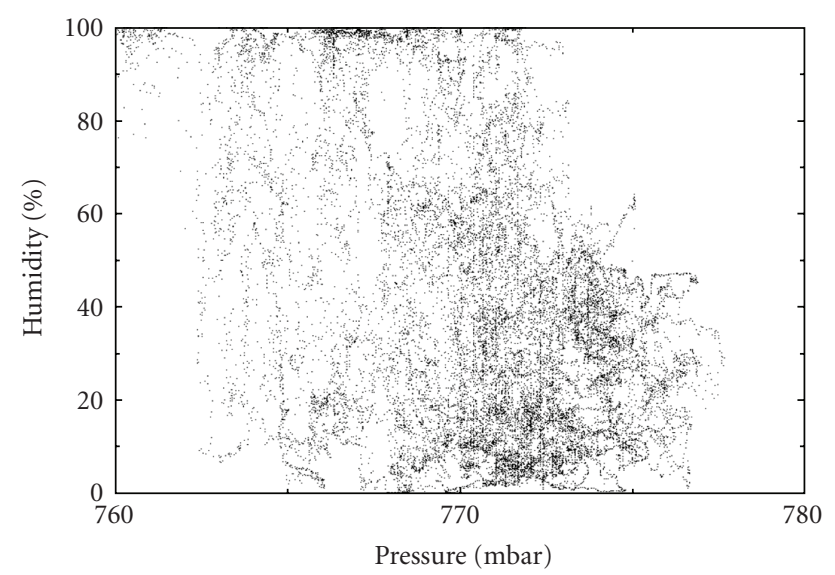

(b)

FIgURE 3: The SCS local cloud predictor. (a) The thick line represents measurements of a sudden humidity increase; that is, a cloud was moving across the STELLA weather stations. The $x$-axis is in seconds and the 2-minute slot needed to close the observatory roof is indicated. The predictor needs in about that time to predict the increase to the actual future value. For the incidence shown this was already the case when the humidity level was still low (50-60\% in this case at $t=10100$ seconds) and the roof closed well before the cloud could have been trapped inside the observatory bay. (b) The basis for that prediction is the (local) relation between humidity and barometric pressure. This relation is not strict but the likeliness of low humidity, that is, good weather, is high when the pressure is also high, that is, when the data fall within the lower right of above diagram.

the distance of the four images produced by the focusing pyramid and the focus position of the telescope was established. This was used to construct a master-focus relation which is just a function of temperature. For bright targets down to 8th magnitude, the optimal focus is determined before the observation starts, for fainter targets the master temperature calibration is applied (see [3] for more details).

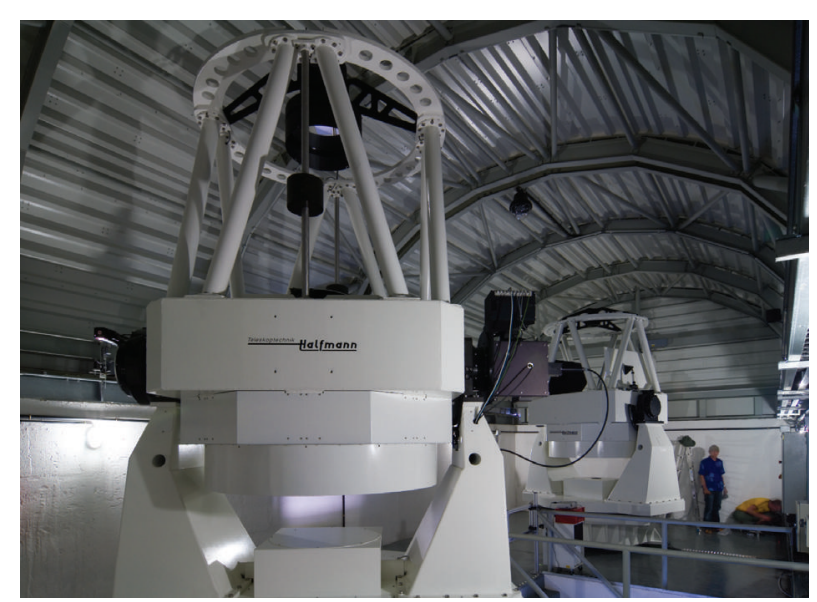

(a)

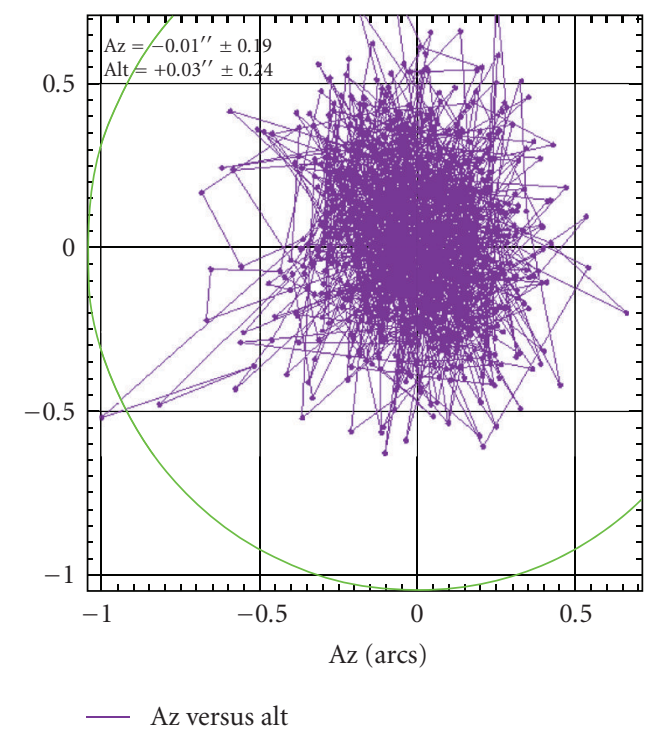

(b)

FIGURE 4: (a) STELLA-I in the foreground with its AG-unit (black box) in the right Nasmyth focus (with the fiber cable making a loop). STELLA-II is in the background. (b) Accumulated guiding corrections for a 1-hour exposure with the SES on STELLA-I. The dots are the individual alt-az pointings. The circle indicates the fiber entrance diaphragm of $120 \mu \mathrm{m}(2.5 \mathrm{arcsec})$. The guiding $\mathrm{rms}$ was 0.19 arcsec in azimuth and 0.24 arcsec in altitude.

At the beginning of routine operation of STELLA-I, fatal failures of the telescope system during the night were rather frequent. It usually meant the loss of the remaining night since recovery after a fatal failure required a hard reset of the telescope electronics which takes its time. Most failures in the first year of observations were caused by such errors. In the meantime, the telescope runs without problems for weeks due to proper error handling, and almost all errors can be treated by the control system in an automated way. Nevertheless, the hard reset capability that we introduced to handle the problems in the beginning, still prove to be useful in solving exceptional problems. 


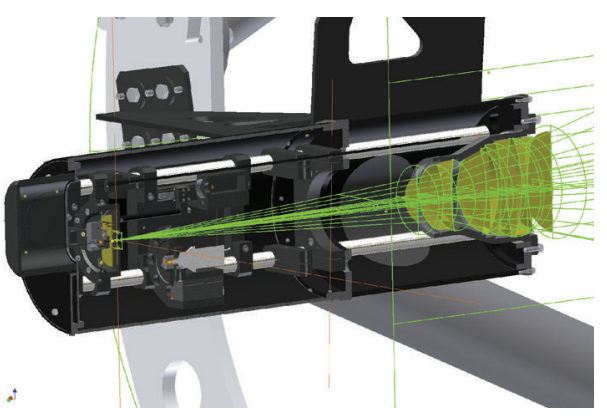

(a)

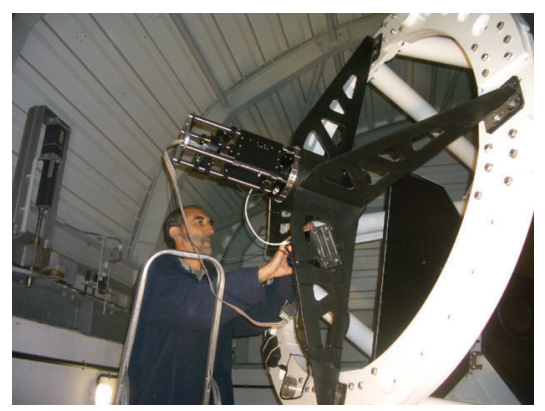

(b)

Figure 5: Design and installation of STELLA-II's prime focus unit. (a) The CAD drawing shows the reflected beam through the fivelens corrector optics (right), an ADC, and a fiber viewing unit. (b) Emil Popow during installation of the unit in the prime focus.

Another important feature is the capability to execute pointing models remotely. The error handler of the SCS realizes when certain regions in the sky experience increased acquisition aborts or even unnatural large offsets. This can be the case when the telescope is out of balance and the azimuth bearing requests higher oil pressure when pointing to a certain region in the sky, for example, always west of the meridian and when the alt position is low. If so, the operator in Potsdam can decide to dedicate a good night to redo the pointing model (see $[6,7]$ and Granzer et al., this proceedings).

STELLA-II is a highly specialized telescope as compared to STELLA-I. Its sole purpose is to feed as much light as possible into a $50 \mu \mathrm{m}$-diameter fiber. This actually does not mean it is a simple telescope. We chose to build the telescope around a space-quality, light-weighted, spherical f/1.95 primary mirror from ZEISS Jena and designed a 5lens prime-focus corrector unit to achieve a PSF matching the fiber entrance f-ratio for optimal injection (see Figure 5). The fiber coupling is done through a microlens based on the EFO-Quartz concept (the microlens is cast and polished by molding the fiber end to a drop). We expect a threefold increase of the light-collection efficiency as compared to the AG-unit on STELLA-I (the current location of the SES fiber until the end of 2009). Acquisition and guiding of the telescopes is currently done with a $150 \mathrm{~mm}$ refracting telescope mounted piggyback to the telescope truss. It includes a $V$-filter and an Audine-based AIP-built guiding camera with a KAF-3200 CCD detector.

\section{STELLA Instrumentation}

\subsection{SES-The STELLA Echelle Spectrograph}

5.1.1. The 2006-2009 Version. The current version of the SES is a white-pupil spectrograph with a fixed wavelength format of $388-882 \mathrm{~nm}$. The instrument is located in a separated, thermally controlled room on a stabilized optical bench (see Figure 6) and is fed with a $15 \mathrm{~m}$-long optical fibre. Currently, a 50 - $\mu \mathrm{m}$ core-diameter fibre enables an instrumental resolution of $\lambda / \Delta \lambda \approx 55,000(0.01 \mathrm{~nm}$ or $\approx 5 \mathrm{~km} / \mathrm{s})$, corresponding to an entrance aperture of $2.1 \mathrm{arcsec}$ on the sky. Its heart is 31 lines per mm R2 grating from RGL. Two off-axis parabolic collimators, one folding mirror, and two prisms as cross-disperser transport the light into the $\mathrm{f} / 2.7$ katadioptric camera with a $20 \mathrm{~cm}$ corrector and a $40 \mathrm{~cm}$ spherical mirror. Beam diameter is $130 \mathrm{~mm}$. The CCD camera is based on a top-grade thinned E2V42-40 device with $2048 \times 2048$ $13.5 \mu \mathrm{m}$ pixels. Its nominal quantum efficiency is $90 \%$ at $650 \mathrm{~nm}$, and $65 \%$ at $400 \mathrm{~nm}$ and $800 \mathrm{~nm}$, respectively, with a full-well capacity well above 100,000 electrons. Together with a second-generation CUO (Copenhagen) controller the read-out noise is 3-4 electrons rms. A closed cycle cooler keeps the detector cooled to $-130^{\circ}$ Celsius. A rotating shutter with a propeller-like blade is located just in front of the CCDentrance window (our "Salami cutter").

The main problem with the current set up is the low overall efficiency $(3 \%)$. This is because we used already pre-existing components from another spectrograph that do not fully cope with the modern instrument design. For example, the Schmidt camera has a folding mirror on the optical axis that is not matched to the fiber exit and thus diffracts up to $40 \%$ of the light. Furthermore, the crossdisperser is red optimized and is rather inefficient short of $400 \mathrm{~nm}$. Its dispersion is also inadequate for the large wavelength coverage which makes strey-light reduction a tedious software issue and will never be perfect (we estimate an average 5\% strey-light contribution which is mostly taken out during the data reduction). The cross-disperser is also not matched to the size of the CCD so that the CCD starts to cut the orders on both ends redwards of $725 \mathrm{~nm}$ and does not leave enough space between adjacent echelle orders. Therefore, an upgrade of the spectrograph was planned all along once the observatory enters routine operation.

5.1.2. The 2010+ Version. Figure 7 summarizes the new SES. The setup remains to be a white-pupil spectrograph with an R2 grating and the two off-axis main and transfer collimators. The Schmidt camera is replaced by an all refractive 7-lens camera with an aperture of $150 \mathrm{~mm}$. It is matched to a thinned and back-illuminated Semiconductor Technology Associates (STA) $4 \mathrm{k} \times 4 \mathrm{k} 15 \mu \mathrm{m}$-pixel CCD. Its controller is a modified AIP-built Magellan controller and enables four-port readout of the device at $\approx 5$ electrons read-out noise in 30 seconds. Its full-well capacity is 70,000 electrons and its cosmetics are excellent. A new crossdisperser will consist of two prisms that optimally distribute the echelle orders across the CCD field of view. The STELLAI AG-unit does not contain an atmospheric dispersion 


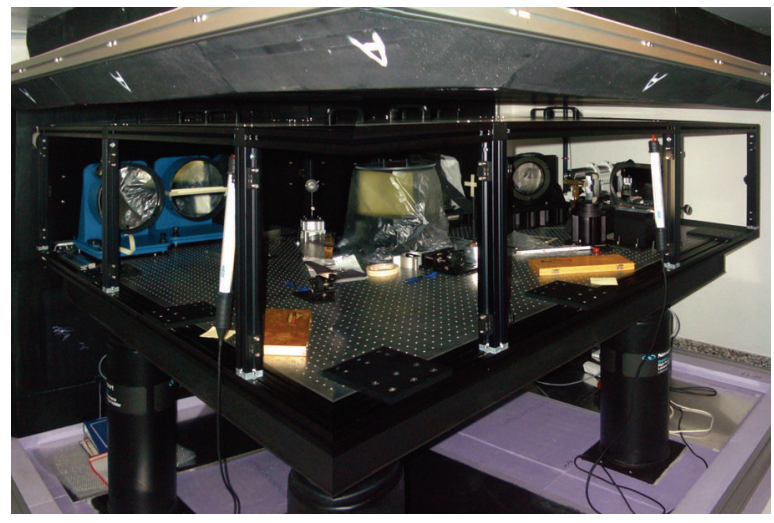

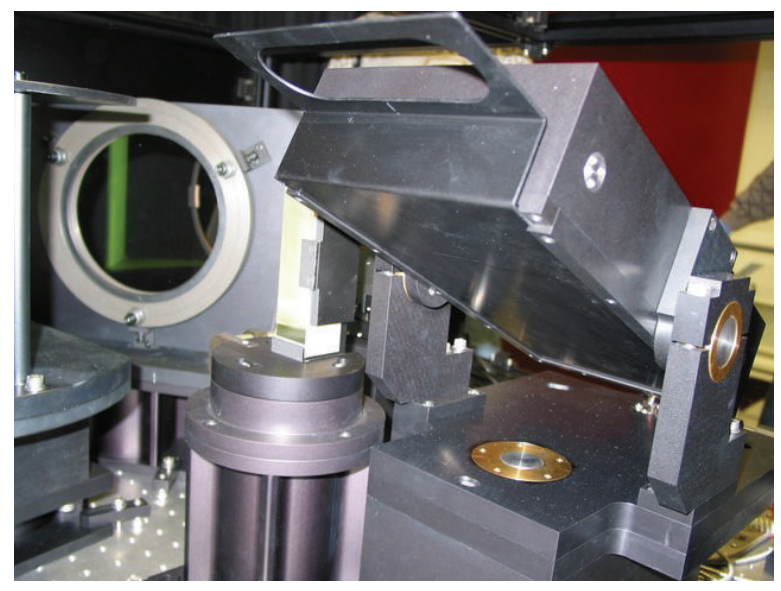

(a)

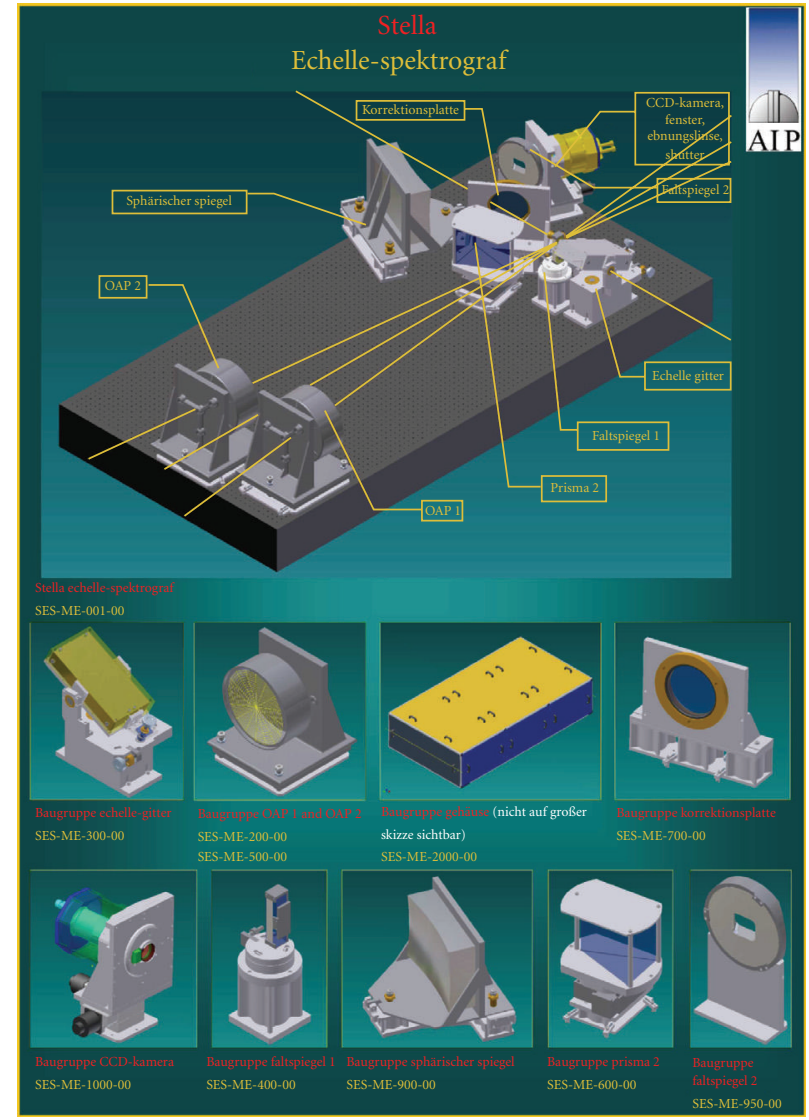

(b)

Figure 6: The STELLA Echelle Spectrograph (SES). (a) top: The instrument is mounted on a Newport optical bench on four gas-damped legs on a pier separated from the building. The table and its optical and mechanical components are enclosed with a dust cover made of anodized aluminium sheets (partly removed in this picture). This cover together with the bench legs is additionally enclosed in a thermal insulation chamber (also partly removed). This chamber is in a dedicated room with active temperature control. (a) bottom: The heart of the spectrograph, the R2 31-1/mm echelle grating (covered). (b) Overview of the opto-mechanical design and its components.

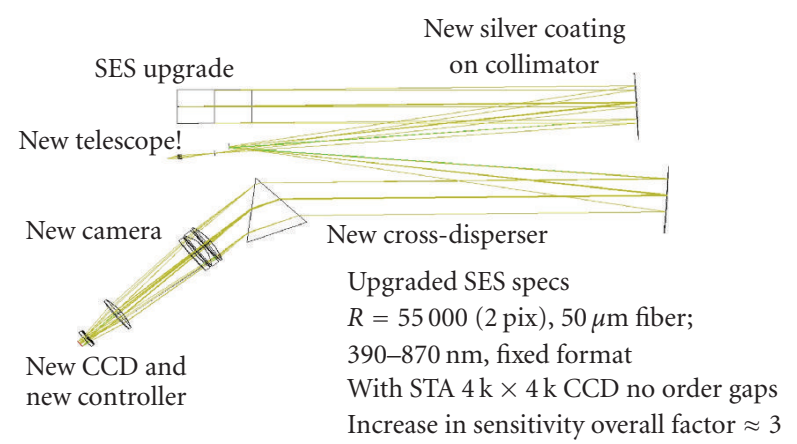

FIGURE 7: Layout of the 2010+ version of the SES. The fiber connection remains to be based on a $50 \mu \mathrm{m}$ Polymicro fiber and the EFO-Quartz principle. The resolution and wavelength coverage remain, the latter without any gaps due to the larger CCD and new cross-disperser. The overall efficiency shall be increased by a factor of three to $\approx 10 \%$ (all inclusive) compared to the current SES version.

corrector (ADC) which added extra losses in the extreme blue and red ends of the spectrum. This will be compensated for in the STELLA-II prime-focus unit. A novel feature of the upgrade will be the simultaneous light feed from a (first) Th-Ar cathode lamp that will be replaced later by a Fabry-Perot halogen lamp. This enables ultra-precise wavelength calibration approaching a radial-velocity stability of (hopefully) few $\mathrm{m} / \mathrm{s}$ without the insertion of an iodine cell that basically ruins the spectrum for other purposes than velocity measurements. 


\subsection{WiFSIP_The Wide-Field STELLA Imaging Photometer.} WiFSIP is a CCD photometer and imager with off-axis guiding. The CCD is a four-port $4096 \times 409615 \mu \mathrm{m}$-pixel back-illuminated CCD from STA and was thinned and antireflection coated at Steward Imaging Technology Lab. It is a twin system of the upgraded SES CCD version. Its controller is optimized for speed though and its current readout noise is 15 electrons rms in 12 seconds read-out time per port. This is sufficient for high-precision photometry. The instrument is currently mounted on RoboTel (see Section 4 and Figure 8) and is being prepared for deployment to Tenerife in late 2009.

The field of view with a 3-lens field corrector on STELLAI is an unvignetted $22 \times 22 \mathrm{arcmin}$ with a scale of $0.32^{\prime \prime} /$ pixel (see Figure 9). The photometer is equipped with $90 \mathrm{~mm}$ sets of Strömgren-uvby, narrow and wide $\mathrm{H} \alpha$ and $\mathrm{H} \beta$, JohnsonBessell UBVRI, and Sloan ugriz filters. A high-speed, doublebladed "Bonn shutter" enables a vibration-free 10 milliseconds exposure with still photometric precision due to counterbalancing the blade's motion. The telescope's limiting magnitude is well below 20th magnitude while it will be difficult to image anything brighter than 10th magnitude due to CCD saturation unless significant telescope defocus. We expect sub-mmag precision for defocused time-series photometry down to 13th magnitude. However, external error sources like telescope jitter are still to be determined and properly handled (robotically of course). Other issues are the low-light level behaviour of the CCD, its quadrant photon mirroring due to the four amplifier architecture of the chip, its (radio) signal pick up suppression, and other telescope interface issues.

\section{Data Handling}

We transfer all raw data including the acquisition frames, the stacked guider frames, and the guiding offsets plus the environmental and status database to the STELLA communication center in Potsdam. Currently, we accumulate about $500 \mathrm{MB}$ of compressed data each day from the SES plus the database contents, which requires an average data rate of $50 \mathrm{kbit} / \mathrm{s}$. An SQL database acts as the central storage pool. Once WiFSIP is operable the data rate will increase at least tenfold due to the much higher cycle time for photometry compared to spectroscopy. Currently, only the SES calibration and data-reduction pipeline are in operation.

6.1. The SES Data Pipeline. The automatic data-reduction pipeline for SES is based on the NOAO Image Reduction and Analysis Facility (IRAF). It consists of a number of IRAF-CL scripts which are invoked by a master script (for more details see [3]). The reduction process is started automatically after the morning calibration exposures are finished and the raw data transferred to Potsdam. The first and largest part contains the bad-pixel correction, bias subtraction, scatteredlight subtraction, cosmic-ray correction, flat fielding, and aperture extraction. Because the orders from about $600 \mathrm{~nm}$ and shorter overlap, we use the program HAMSCAT [8] to fit the scattered light and the light pollution from adjacent

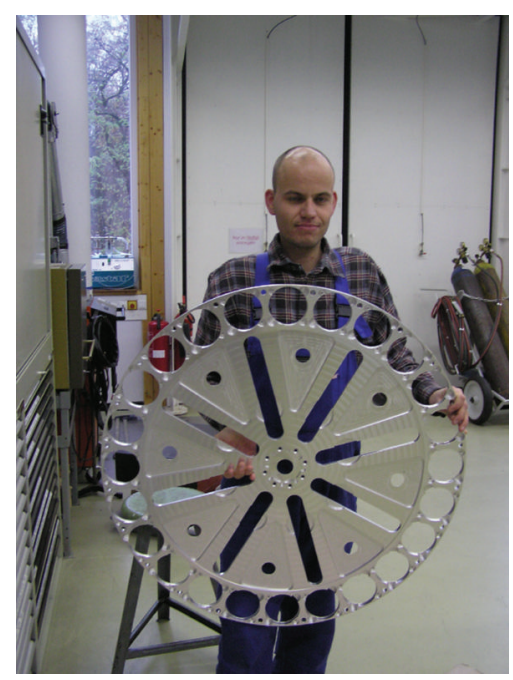

(a)

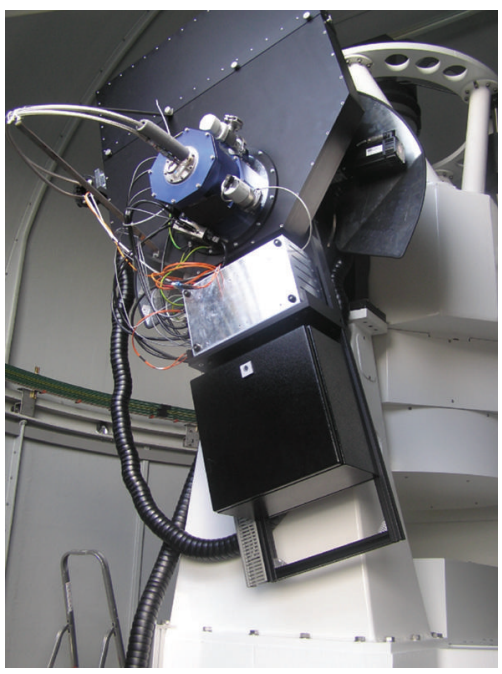

(b)

FIgure 8: The Wide-Field STELLA Imaging Photometer WiFSIP. (a) The large 24-hole filter wheel during manufacture in the AIP workshop. (b) The instrument during first tests mounted on the $0.8 \mathrm{~m}$ RoboTel Nasmyth focus. The box with the filter wheel is now located on the top with the CCD dewar in blue connected to a closed-cycle cryocooler (not shown). The middle box contains the CCD controller in a thermally insulated aluminium enclosure. The lower black box contains the remaining electronics. Note that the instrument is flanged to an image derotator and is turning to compensate for the field rotation due to the alt-az mount.

orders and remove it. Order extraction is done using IRAF's optimal extraction. The current wavelength calibration uses a predefined Th-Ar line list and interpolates between two Th-Ar exposures closest in time before and after the science observation. The raw FITS images along with the end products of the data reduction pipeline are then moved to a backup server. The second part consists of continuum fitting which is currently not automated but will be once the SES upgrade is installed (see Figure 1 for a spectrum example). 


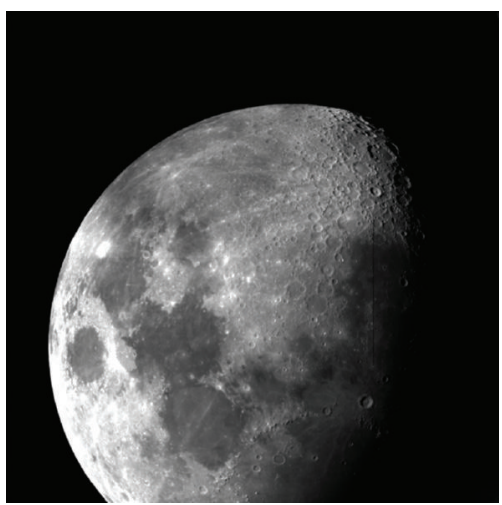

Figure 9: The WiFSIP CCD field of view on RoboTel $\left(30^{\prime} \times 30^{\prime}\right)$.

Once the spectra are reduced and properly back upped, an automated analysis of the reduced spectra is launched. It currently includes radial velocity measurements with both a template spectrum computed from a model atmosphere appropriate for the star in question and with a radial velocity standard star spectrum. Template spectra are tabulated with metallicities between $-1 \mathrm{dex}$ and $+0.5 \mathrm{dex}$ in steps of $0.5 \mathrm{dex}$, logarithmic gravities between 0 and 5 in steps of 0.5 , and temperatures between $3500 \mathrm{~K}$ and $50,000 \mathrm{~K}$ in steps of $250 \mathrm{~K}$ for a wavelength range of $380-920 \mathrm{~nm}$. The user can choose the velocity that gives the most consistent result for his/her particular type of target. The next and final step is the determination of the best-fit effective temperature $T_{\text {eff, gravity }} \log g$, and metallicity $[\mathrm{Fe} / \mathrm{H}]$ of the target star. This is done with the program PARSES [9] by fitting atmospheric models to the spectral region containing $\mathrm{H} \beta$. For this purpose, the SES spectra are degraded to a resolution of $\approx 7,000$ because PARSES in its current version neglects rotational line broadening. An update will be implemented soon and then the full resolution in five spectral orders will be used.

6.2. The WiFSIP Data Pipeline. As for the spectrograph, data reduction for WiFSIP will be done in Potsdam once the raw data has been transferred. For flat fielding of the science data, sky flats are taken at morning and evening twilight. The high number of available filters inhibits a procedure where flats for all filters are taken during twilight. Thus, during twilight only flats for a single photometric set (Johnson, Strömgren, or Sloan) are taken. The SCS tries to anticipate within the evening twilight which filter set is the one with the most urgent need, depending on flat history, flat stability, and filters to be used in the upcoming night, a decision that could be refined later during dawn twilight. Once all data has been transferred to the AIP, a preparatory pipeline assures that for any given night, the most recent bias and flat calibration frames are available. Depending on the secured flat-field stability, a warning is issued if the flats in proximity to the night in question are too far off in time in order to assure a pixel-to-pixel stability exceeding $10^{-3}$. The science pipeline itself is based on the ICAT-pipeline ([10]; see also Colomé et al., this proceedings), which, in turn, utilizes NOAO-IRAF for bias and dark subtraction and flat fielding. Astrometric calibration is done using WCSTools [11]. For photometry, two methods are available, the aperture-photometry package SExtractor [12], and the PSF-modelling package DAOPHOT [13]. The instrumental magnitudes along with the astrometric data are collected in a database. From there, time-series of ensembles of images can be extracted using a modified version of the ENSEMBLE4 package [14]. The current development focuses on the incorporation of standard stars to gain full-sky absolute photometric solutions.

\section{Early Results}

Currently, STELLA-I/SES carries out 12 programs from German, Spanish, U.S., and Hungarian investigators on a private communication basis and without further quality control. Until the SES is upgraded, we dub this phase the science demonstration time. A few auxiliary programs are highlighted in what follows.

7.1. HD 1-The Number One Star in the Sky. HD $1=$ HIP 422 is among the few rather bright stars $(V=7.4 \mathrm{mag})$ of which we basically know nothing except some very basic parameters. It is thus a well suited target for a data demonstration and we have randomly chosen HD 1 to be our first science verification target. We find the star to have a systematically variable radial velocity and suggest it be a single-lined spectroscopic binary in a very long and eccentric orbit. It appears to be a $\mathrm{K} 2$ bright giant of luminosity class III-II with $\approx 300 \mathrm{~L}_{\text {Sun }}$ at an age of $130 \mathrm{Myr}$, a mass of $4 \pm$ $1 \mathrm{M}_{\text {Sun }}$, and a radius of $\sim 27 \mathrm{R}_{\text {Sun }}$.

7.2. $\varepsilon$ Aurigae Entering Its Next Eclipse after 27 Years. $\varepsilon$ Aur is a binary star system that eclipses once every 27.1 years. The next eclipse is currently ongoing and began in July 2009. The eclipse is flat-bottomed and lasts nearly two years. A mideclipse brightening was seen by several observers, including observations from space, and many models appeared for its explanation. Among them a neutron stars with a warped semiopaque disk. Photometry of the primary F star appears to show pulsational variability with at least eight periods ranging from $>570$ days to 48 days [15]. Our STELLA SES data show short-term radial velocities variations with a peakto-peak amplitude of up to $25 \mathrm{~km} / \mathrm{s}$ consistent with the photometry.

7.3. Capella-Two Active Giants in a 104-Day Orbit. Astrophysical parameters of giant stars are uncertain because there are just a few such stars that can be studied in spectroscopic binary systems. The present position of the G1III component of $\alpha$ Aur in the Hertzsprung gap, where it is approaching the base of the giant branch, indicates that violent changes are taking place in its internal structure; the mass of the convection zone increases rapidly as does also the total stellar moment of inertia while the other component is likely already helium burning. We may expect that this has an impact on the overall magnetic activity of the system. We 


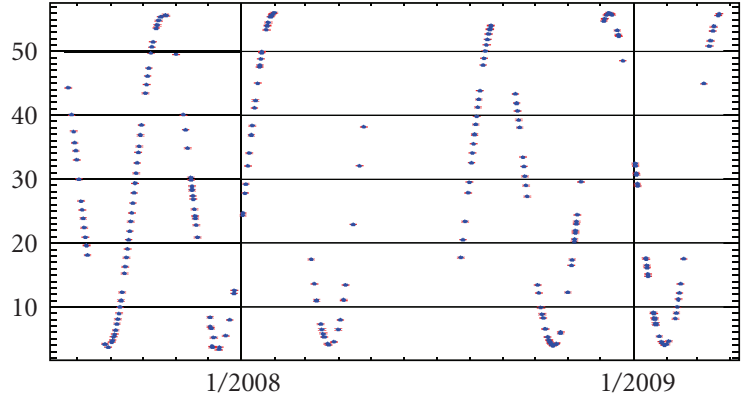

FIgURE 10: Radial velocities for the primary giant of the Capella binary system. Note that the orbital period is 104 days and that the total time coverage is three years. Gaps are mostly due to the observing season but also due to the various weather-related and technical down times.

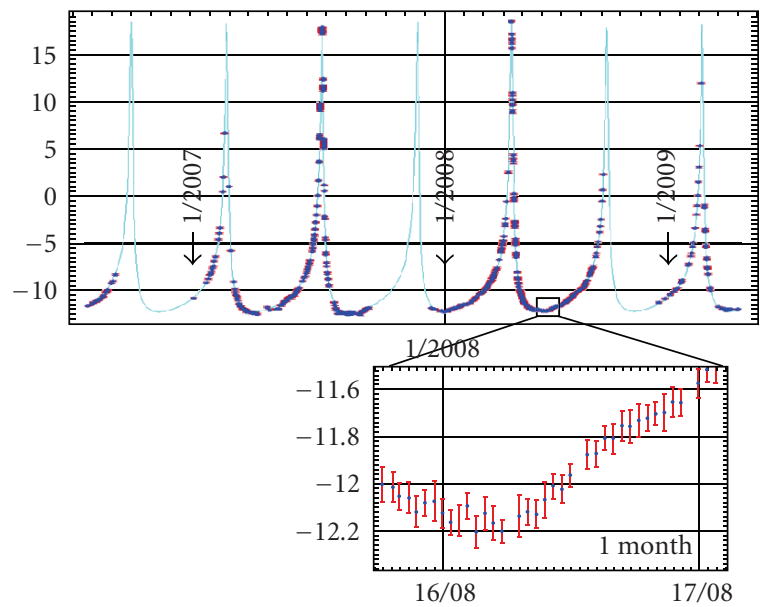

Figure 11: Radial velocities and orbit for HD123351. The star turned out to be a very eccentric single-lined spectroscopic binary with a period of 148 days. Note that its rotational period can be seen in the radial velocities due to the asymmetric distribution of its starspots.

monitor the Capella system with one spectrum every clear night. Its radial velocities from the SES data pipeline are shown in Figure 10.

7.4. The Surprising Radial Velocities of HD123351. Another extreme magnetically active star is HD123531. Known to be a K2IV star it was assumed to be single due to constant radial velocities. Soon after we started monitoring with STELLA-I it turned out to be a binary in a very eccentric 148-day orbit. The eccentricity is 0.81 and the semiamplitude near $15 \mathrm{~km} / \mathrm{s}$. The radial velocities and a preliminary orbit are shown in Figure 11 . Note that the radial velocities are also rotationally modulated!

7.5. The Exoplanet 51 Pegasi b. For demonstration purposes we also monitored the famous exoplanet system 51 Pegasi. Figure 12 shows the radial velocities and a clear detection of the star's hot Jupiter planet. The overall rms of the data is

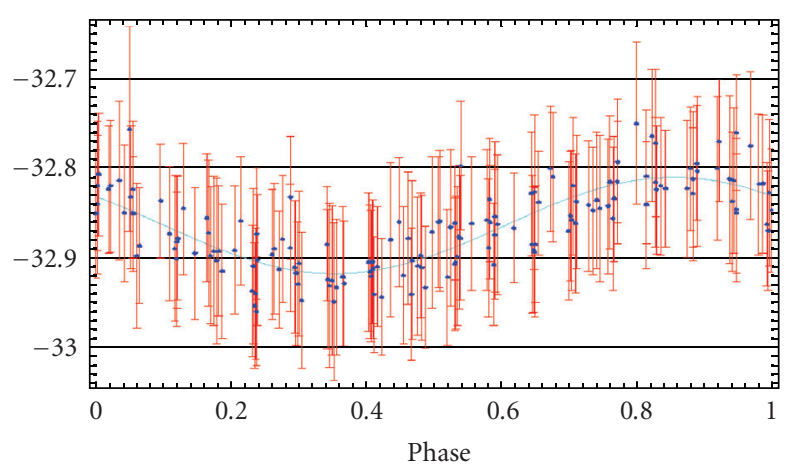

Figure 12: The planet of 51 Pegasi with STELLA-I/SES. The orbital period of the planet is 4.23 days ( $e=0$; continuous line) and the velocity amplitude is $\approx 100 \mathrm{~m} / \mathrm{s}$. Our overall $\mathrm{rms}$ of the data is $30 \mathrm{~m} / \mathrm{s}$, an individual measurement error is $\approx 70 \mathrm{~m} / \mathrm{s}$. The data span between May and August in 2008 and are plotted directly from the SES pipeline. Note that no other measures than the thermal and mechanical stabilization of the spectrograph were taken.

$30 \mathrm{~m} / \mathrm{s}$. Note that no iodine cell or simultaneous Th-Ar light or template cross correlation is involved.

7.6. Doppler Imaging of FK Comae. Early on, we participated in a simultaneous observing campaign with ESO's VLT to map the magnetic field of the active rapidly rotating giant FK Comae. The STELLA/SES data were used to obtain a Doppler image from an inversion of its photospheric line profiles ([16]; see Figure 13). Simultaneously, a dynamic spectrum of the accompanying $\mathrm{H} \alpha$ line profiles suggested a close relation with the photospheric spots and the phases of strongest magnetic field.

7.7. Beteigeuze's Convective Motion Pattern. Hydrodynamic "star-in-a-box" simulations show Beteigeuze with a very course and time-variable granulation pattern [17]. Roughly 20 granulation cells at a time are covering this $800-\mathrm{R}_{\text {Sun }}$ $\mathrm{M}$ supergiant with a typical life time of a "few years." We monitor the star's radial velocities with high precision in order to detect the granulation signature and to compare it with numerical model predictions.

7.8. Active Star Binary Orbits. Magnetically very active stars in binaries are thought to modulate the orbital period due a feedback on the gravitational quadrupole moment from a magnetic activity cycle induced rearrangement of internal angular momentum. STELLA-I/SES monitors approximately 50 active stars, most of them previously unknown binaries.

7.9. Gliese 586A-Monitoring the Most Eccentric Binary Orbit Known. Two cool M dwarfs revolve around a common center of mass with a period of 890 days and a record eccentricity of 0.975 [18]. Despite the long period, the periastron where the radial velocity reverses its sign is passed in just 24 hours and spans $70 \mathrm{~km} / \mathrm{s}$ at $5 \mathrm{~km} / \mathrm{s}$ per hour. So far, STELLA covered one periastron passage in 2007 but the next passage is coming up in 2009. Figure 14 shows the current status of the 

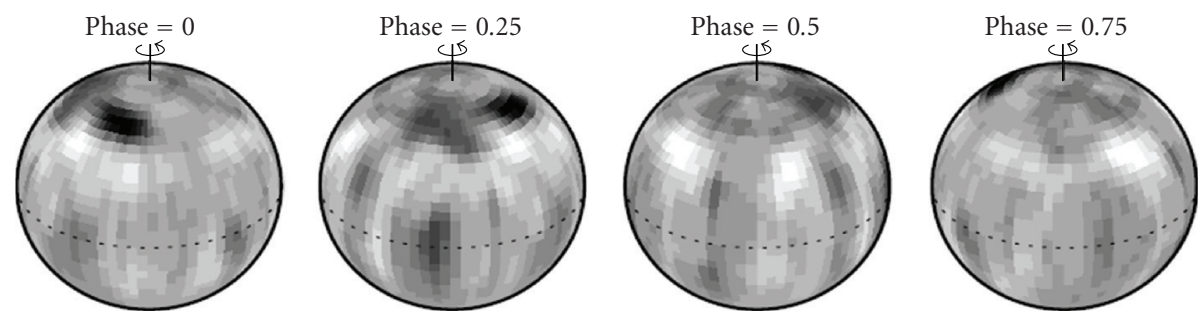

$(\mathrm{K})$

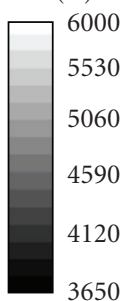

FIGURE 13: Doppler image of the rapidly rotating G giant FK Comae based on STELLA-I SES data. The map plots surface temperature according to the scale. It was taken from [16].

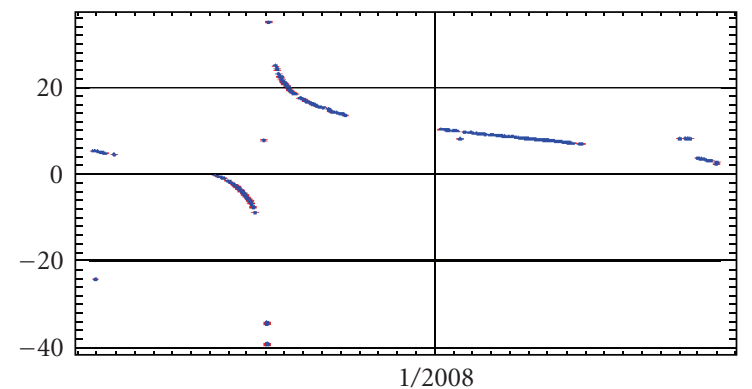

FIGURE 14: Radial velocities in $\mathrm{km} / \mathrm{s}$ of Gliese 586A. The time coverage in this plot is $\approx 900$ days, that is, one full orbital period of Gl586A. The gaps are the seasons when the target is unobservable. Also note some misidentifications at a constant velocity of $8 \mathrm{~km} / \mathrm{s}$ (likely one of the other visual binary components, Gliese $586 \mathrm{C}$ or $\mathrm{D} ; t b d)$.

monitoring program. Only the brighter of the two M dwarfs is shown in this preliminary radial-velocity plot.

\section{Acknowledgments}

STELLA is funded by AIP through the State of Brandenburg and the Federal Ministry for Education and Science in Germany. It is operated jointly by AIP and the Instituto de Astrofisica de Canarias (IAC) at the Teide Observatory of the IAC. The authors thank the staff of the IAC for their great and continuous support to run STELLA.

\section{References}

[1] K. G. Strassmeier, T. Granzer, M. Weber, et al., "The STELLA robotic observatory," Astronomische Nachrichten, vol. 325, no. 6-8, pp. 527-532, 2004.

[2] K. G. Strassmeier, T. Granzer, M. Weber, et al., "STELLA: two new robotic telescopes for binary-star research," Astrophysics and Space Science, vol. 304, no. 1-4, pp. 397-400, 2006.

[3] M. Weber, T. Granzer, K. G. Strassmeier, and M. Woche, "The STELLA robotic observatory: first two years of high-resolution spectroscopy," in Advanced Software and Control for Astronomy II, vol. 7019 of Proceedings of SPIE, Marseille, France, June 2008.

[4] T. Granzer, "Generic control of robotic telescopes," in Advanced Software, Control, and Communication Systems for
Astronomy, vol. 5496 of Proceedings of SPIE, pp. 667-678, Glasgow, UK, June 2004.

[5] T. Granzer, M. Weber, and K. G. Strassmeier, "Automation hardware and software for the STELLA-I robotic telescope," Astronomische Nachrichten, vol. 322, no. 5-6, pp. 295-302, 2001.

[6] T. Granzer, "What makes an automated telescope robotic?" Astronomische Nachrichten, vol. 325, no. 6-8, pp. 513-518, 2004.

[7] K. G. Strassmeier, K. Agabi, L. Agnoletto, et al., "Telescope and instrument robotization at Dome C," Astronomische Nachrichten, vol. 328, no. 6, pp. 451-474, 2007.

[8] C. W. Churchill and S. L. Allen, "A treatment for background correction on the Hamilton Echelle Spectrograph," Publications of the Astronomical Society of the Pacific, vol. 107, no. 708, pp. 193-198, 1995.

[9] C. Allende Prieto, "Automated analysis of stellar spectra," Astronomische Nachrichten, vol. 325, no. 6-8, pp. 604-609, 2004.

[10] J. Colomé and I. Ribas, "ICAT: a general purpose image reduction and analysis tool for robotic observatories," in Astronomical Data Management, 26th meeting of the IAU, Special Session 6, Prague, Czech Republic, August, 2006, SPS6, $\# 11$.

[11] D. Mink, "WCSTools 4.0: building astrometry and catalogs into pipelines," in Astronomical Data Analysis Software and Systems XV, C. Gabriel, Ch. Arviset, D. Ponz, and E. Solano, Eds., vol. 351 of Astronomical Society of the Pacific Conference Series, p. 204, 2006.

[12] E. Bertin and S. Arnouts, "SExtractor: software for source extraction," Astronomy and Astrophysics Supplement, vol. 117, pp. 393-404, 1996.

[13] P. B. Stetson, "DAOPHOT-a computer program for crowded-field stellar photometry," Publications of the Astronomical Society of the Pacific, vol. 99, pp. 191-222, 1987.

[14] E. Distefano, S. Messina, G. Cutispoto, et al., "ARCO: a program for automatic reduction of CCD observations," N. Epchtein, et al., Ed., vol. 25 of EAS Publications Series, pp. 165169, Roscoff, France, 2007.

[15] K. G. Strassmeier, P. Reegen, and T. Granzer, "On the rotation period of Capella," Astronomische Nachrichten, vol. 322, no. 2, pp. 115-124, 2001.

[16] H. Korhonen, S. Hubrig, S. V. Berdyugina, et al., "First measurement of the magnetic field on FK Com and its relation to the contemporaneous star-spot locations," Monthly Notices of the Royal Astronomical Society, vol. 395, no. 1, pp. 282-289, 2009. 
[17] B. Freytag, M. Steffen, and B. Dorch, "Spots on the surface of Betelgeuse-results from new 3D stellar convection models," Astronomische Nachrichten, vol. 323, no. 3-4, pp. 213-219, 2002.

[18] A. Duquennoy, M. Mayor, J. Andersen, J.-M. Carquillat, and P. North, "Duplicity in the solar neighbourhood. VI. The highly eccentric spectroscopic binary Gl 586A and associated multiple system," Astronomy and Astrophysics, vol. 254, no. 1, pp. L13-L16, 1992. 

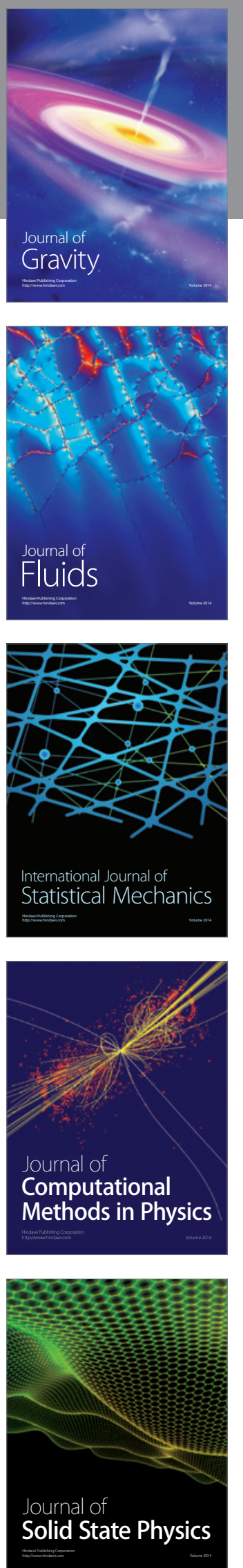

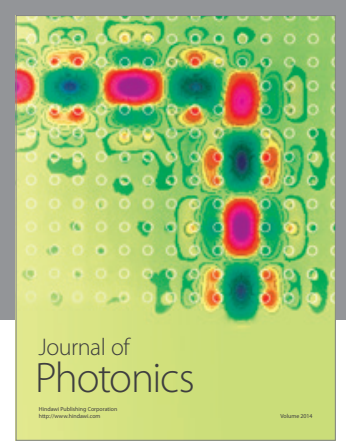

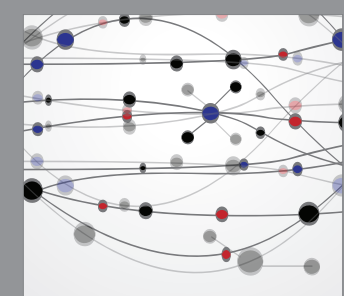

The Scientific World Journal
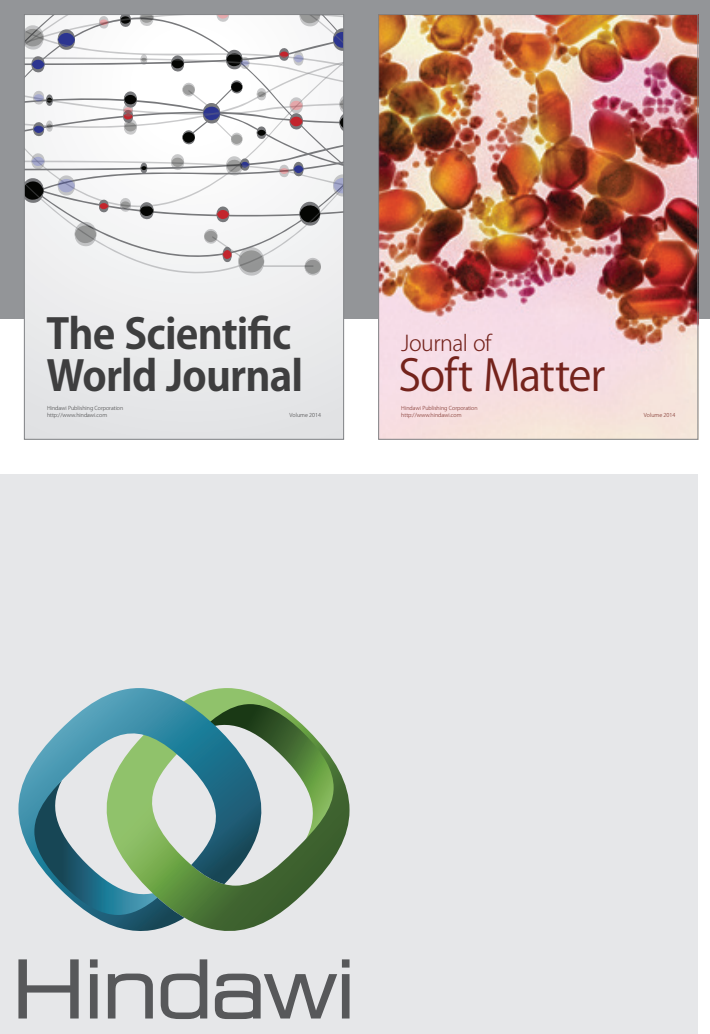

Submit your manuscripts at

http://www.hindawi.com
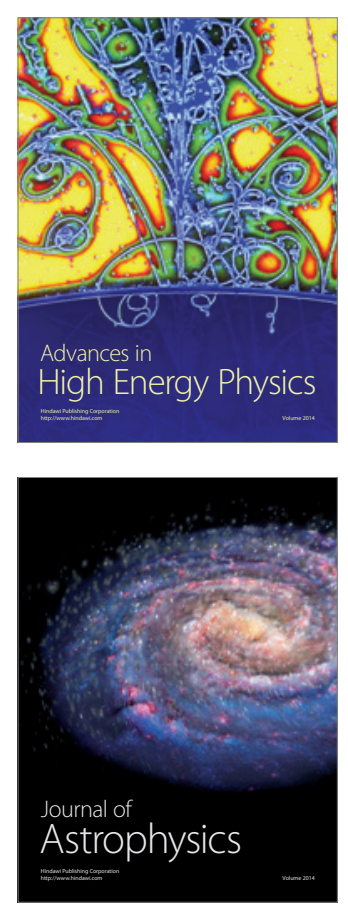
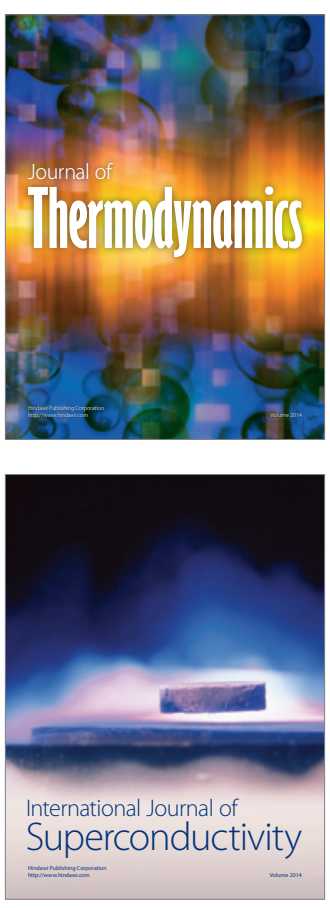
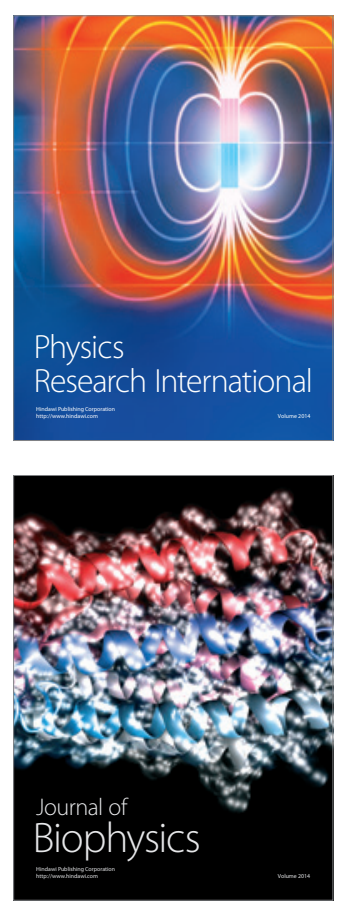
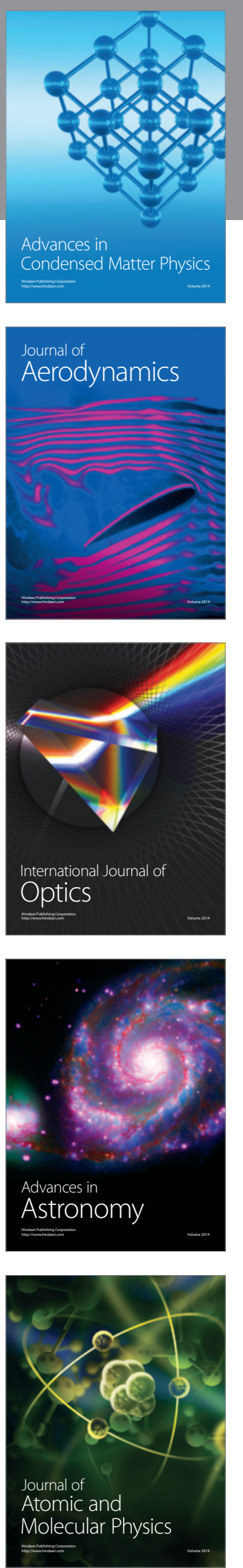